\title{
Inspection of Mechanical Tolerance by Using the Virtual Gauge on a Coordinate Measuring Machine
}

\author{
Salim Boukebbab ${ }^{1, *}$, Jean M. Linares ${ }^{2}$, Med S. Boulahlib ${ }^{1}$ and Jean M. Sprauel ${ }^{2}$ \\ ${ }^{1}$ Laboratory of Mechanics, Faculty of Engineering Sciences, Mentouri-Constantine University, Road of Ain-el-Bey, \\ 25000 Constantine, Algeria, ${ }^{2}$ Laboratory of EA(MS), Mediterranean University, Technology Institute University, Road \\ of Gaston Berger, 13625 Aix-en-Provence Cedex 1, France
}

\begin{abstract}
The function to obtain associated surfaces on coordinate measuring machines (CMM's) is based on the minimization of the distance between measured points and the ideal surface. This function is non-linear for usual surfaces. In many works, to accelerate iterative calculations, the problem is linearized. The aim of this work is to reduce scraps using a transcription optimization of the fitting functionality of mechanical parts in maximum state of matter. An adaptive verification method is suggested. It takes account of the interface properties. A control by a virtual gauge and verification a process is developed to validate the tolerancing according to the previously suggested methodology.
\end{abstract}

Keywords: Virtual gauge, metrology, fitting, no linear method, optimization, least squares method, coordinate measuring machine.

\section{INTRODUCTION}

A machine, such as a coordinate measuring machine (CMM's), uses a material measure with a periodic division in order to determine spatial positions of a working or measuring head [1]. The principle of the CMM's software consists in individually associating an elementary mathematical model (plane, cylinder, etc) to each acquisition surface [2]. The function to be minimized is based on the distance $y_{i}$ between the digitized point $M_{i}$ and the theoretical surface. The result differs according to the chosen minimization criterion (least square, Tchebichev criterion, minimax...) [3-6]. This function is non-linear for usual surfaces (line in space, plane surface, cylinder, cone and sphere). To reduce the computing time of the optimization procedures, many works have approximated the displacement matrix to the first order [7,8], and most metrology software packages use this method to calculate the parameters of the associated surface. Nowadays, an apparatus and method for automatically identifying faults in the operation of a machine vision measuring systems provides an improved self-diagnostic capability for machine vision based metrology and tracking systems $[9,10]$.

The fitting of theoretical surfaces to a set of points consists in defining an initial position close to the optimum position so that the small displacements screw method can be used. The assumptions of this method require that the displacements are small and that the measuring surface has a low form error in comparison with the geometric error (orientation, position.).

In this paper, a non linear method is used. From the model of tolerancing proposed, it is possible to create a

\footnotetext{
*Address correspondence to this author at the Laboratory of Mechanics, Faculty of Engineering Sciences, Mentouri-Constantine University, Road of Ain-el-Bey, 25000 Constantine, Algeria; Tel/Fax: 0021331818853 / 63: E-mail: boukebbab@yahoo.fr
}

virtual gauge for a better transcription of the functionality. This gauge represents the connecting interface of the part during the assembly of the mechanism.

\section{METHODS OF TOLERANCING TOOLS AND THEIR IMPLICATIONS}

In the tolerancing phase, the designer has tolerancing tools (straightness, flatness, perpendicularity, position...) and principles (maximum material requirement, envelope requirement ...). According to its choices, he can define the verification procedures of the toleranced parts in advance.

The envelope requirement applies in the many cases for which an assembly function is needed. The assembly (fitting) function is indeed transcribed by the requirement for a maximum material condition. The tolerancing with the maximum material condition may be classified in two types Fig. (1):

a) Type I: Transfer of the diameter variation onto the geometric tolerance when the surfaces are in the maximum material virtual state. The datum surface and specified surface are the dimensional resources.

b) Type II: Transfer of the diameter variation of the datum system onto the geometric tolerance

Tolerancing based on requirements directs the inspection towards the verification by a gauge $[11,12]$. It reconstructs the functional environment present in the assembled mechanism. The cost generated by the manufacturing of materiel gauges entails the suggestion to use virtual gauges based on computer simulation. The inspection is a binary process [13]. However some limits concerning the use of the maximum material condition can be noted here; when the type II is used is used, because no formulation exits at the level of design and verification that allows managing dimensional transfers onto the geometric tolerance [14]. 
a)

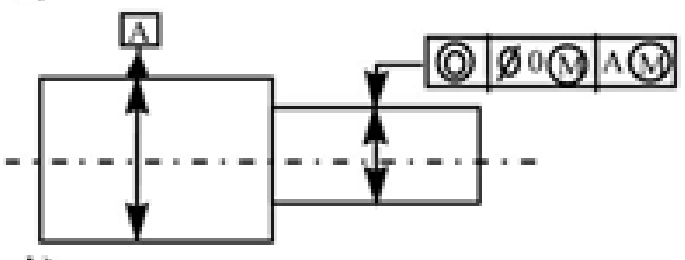

b)

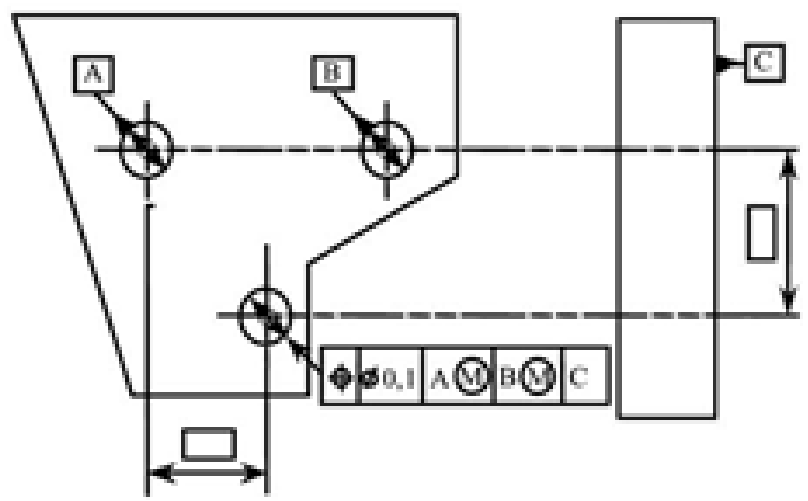

Fig. (1). Example for tolerancing with the maximum material condition, a) type I, b) type II.

Moreover, in the most recent releases of Coordinate Measuring Machines software, only the cases of type I transfers are implemented. The interfaces between two groups of surfaces for assembly often show freedom potentialities (clearance and variation of internal tolerancing). Neglecting the clearances in these interfaces results in increasing constrains of functional tolerancing. To conclude this paragraph we can show the existing relations between design and control according to the use of requirements Fig. (2).

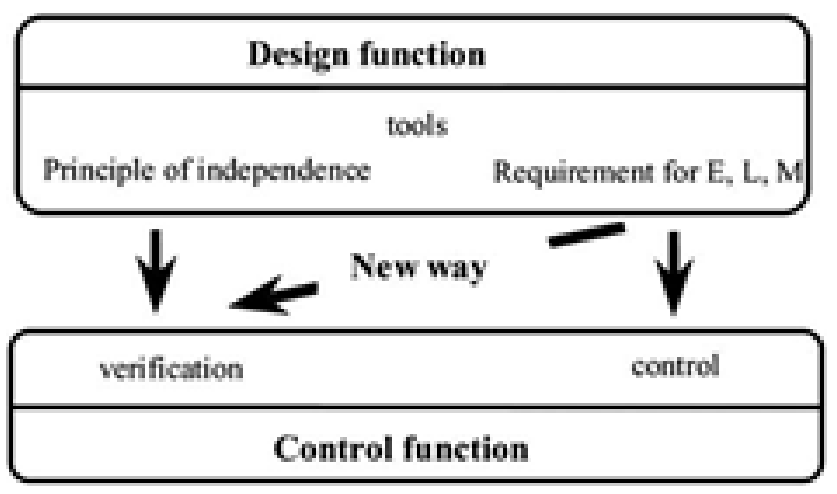

Fig. (2). Relation between design and control.

This illustration points up some failures in industry when physical gauges are replaced by a coordinate measuring machines which basically performs rather measurement then control in the present state of software. In [15] Methods for determining tolerances are disclosed that can be used for determining whether a lot of semiconductor wafers needs to be reworked.

\section{EXAMPLE OF THREE-DIMENSIONAL TOLE- RANCING}

To illustrate the management of dimensional transfers onto the geometric tolerance, the example presented in Fig. (3) is used.

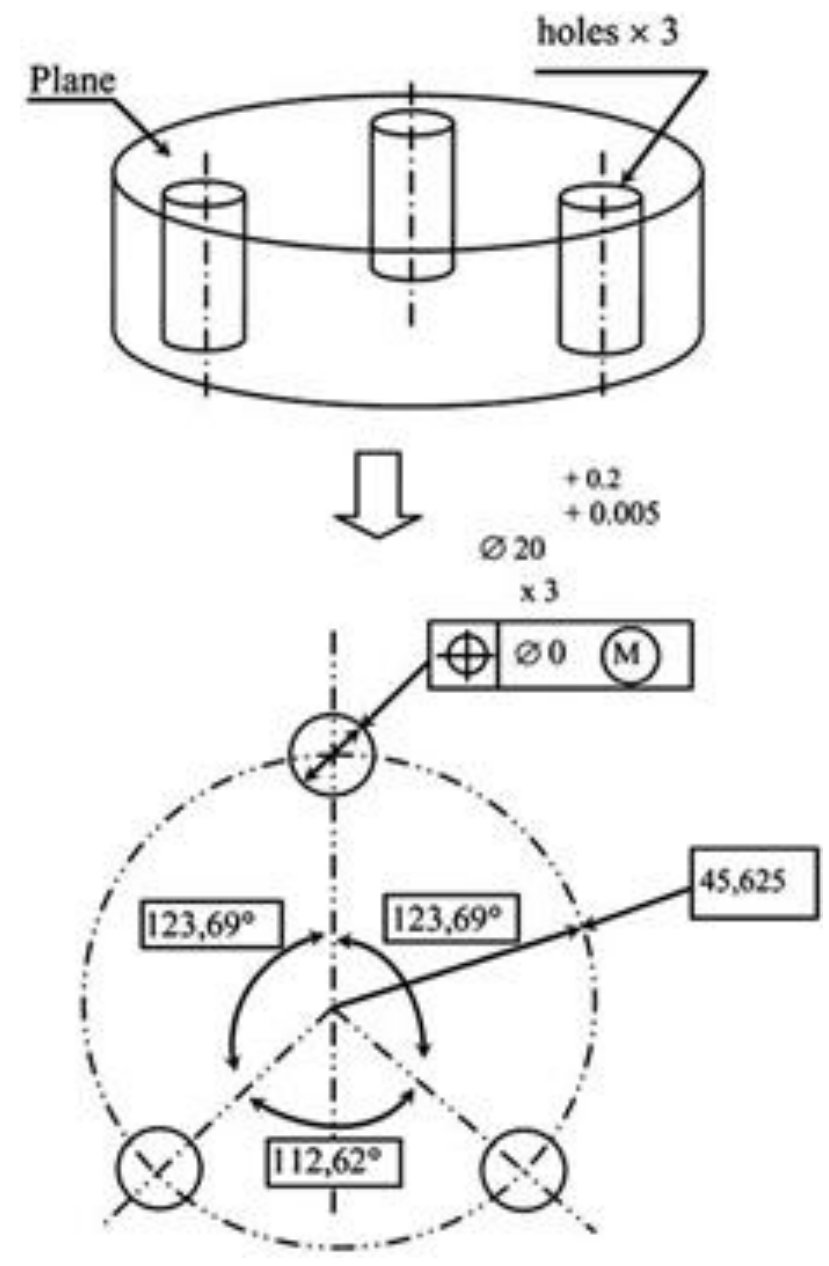

Fig. (3). Tolerancing suggested.

This specification can be classified in type II, because the three holes are specified in maximum state of matter. The conformity of the latter will be established by the verification process. A methodology for the control of this specification is proposed.

\section{CONTROL FUNCTION}

The inputs required for the verification procedure are files containing all the points $\mathrm{M}_{\mathrm{i}}$ of the measured surfaces digitized in the measurement coordinate system and the parameters of the geometric specifications.

The vectors and the centers of the surfaces fitted to theses points are first determined, in the measurement coordinate system after minimization of the deviations $y_{i}$ between the digitized coordinates and the perfect geometric element Fig. (4). 


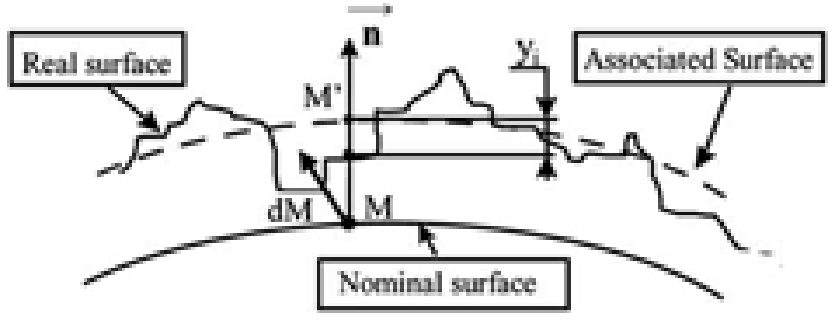

Fig. (4). Modeling of real surfaces.

The attributes of the fitted surfaces are finally obtained through a non linear least squares optimization [16].

\subsection{The Large Displacements Method}

As already pointed out, the actual coordinate measuring machine software's are based on an approximation of the deviations by a linear first order expansion. For that purpose each point of the ideal surface to be fitted to the acquired coordinates is moved iteratively from an initial position $\mathrm{M}$ to a transformed one $\mathbf{M}^{\prime}$, in order to reduce the deviations $\mathrm{y}_{\mathrm{i}}$ Fig. (4). This displacement is obtained by three translations and three rotations. If it remains small, the displacement

$\mathrm{dM}=\mathrm{MM}^{\prime}=\mathrm{OM}^{\prime}$ OM can be modulated by a small displacement screw [17].

To satisfy this assumption, it is then necessary to move in a local co-ordinate system. This type of calculation requires as first step that the software estimates a position of the fitted surface close to the final solution. Then the optimization algorithm minimizes the "error function $\mathrm{y}_{\mathrm{i}}$ " in several iterations. The result is then known in a local coordinate system. An inverse transformation (local co-ordinate system to global reference frame) gives the parameters of associated surface in the global reference frame [18]. The advantage of this method is that the equation to be minimized is easy to find for the matrix operation becomes a vector operation and the function is linear. The linearity of this equation allows obtaining $\mathrm{y}_{\mathrm{i}}$, with a simplified optimization algorithm. In the seventies this characteristic had the advantage of reducing the calculation time of less advanced computers [19].

With the non linear method, the assumptions of infinitely small rotation angles and small displacement are no longer necessary. In our case, the equation of $\mathrm{y}_{\mathrm{i}}$ is non linear but it offers the advantage of giving the results after optimization in the global co-ordinate system, and the calculated distance $\mathrm{y}_{\mathrm{i}}$ does not undergo any approximation.

In the continuation of this article, the large displacement method will be used to model three cylindrical surfaces (holes) and a plane. Their virtual gauge will be constructed to test the conformity of the geometrical specification given in Fig. (3).

\subsubsection{Modeling of Cylindrical Surfaces}

Each measured point $\mathrm{M}_{\mathrm{i}}$ with the coordinates $\left(\mathrm{x}_{\mathrm{j}}\right)_{\mathrm{j}=1 \mathrm{to} 3}$ is known in the coordinate system of the CMM's Fig. (5). The $\mathrm{y}_{\mathrm{i}}$ equation becomes:

$y_{i}=\mathrm{CM}_{i} 1 \mathrm{v} R$

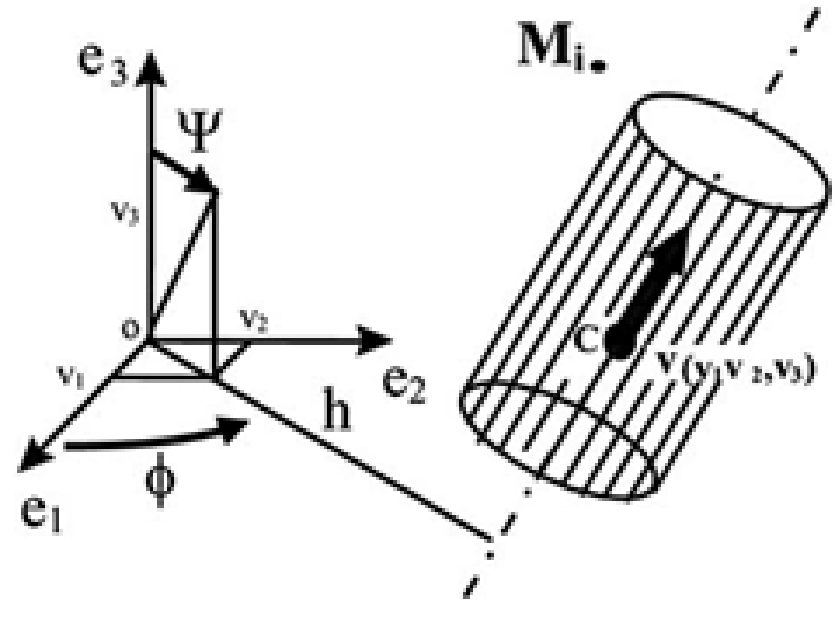

Fig. (5). Calculation of the distance $y_{i}$.

With $\mathbf{v}$ being the direction vector of the cylinder axis, $\mathrm{C}$ its characteristic point and $\mathrm{R}$ the radius of the cylinder.

To impose the normality condition of the direction vector $\mathbf{n}$, this vector is defined in the cylinder coordinate system introducing two angles $(, 1)$. The centre $\mathrm{C}$ of the axis is obtained by the projection of the centre of gravity of the measured coordinates to this axis. All the parameters are known in the global coordinate system $\mathrm{R}_{\mathrm{o}}\left(0, \mathrm{e}_{1}, \mathrm{e}_{2}, \mathrm{e}_{3}\right)$. In this non-linear method, the distance $y_{i}$ is not subjected to any approximation.

The optimization according to the least squares method enables us to identify the attributes of the fitted surfaces thanks to the following relation:

$$
{\frac{1=1}{a} y_{i}^{m}}_{i}=0
$$

With i: number of digitized points

$\mathrm{a}_{\mathrm{j}}$ are the optimization parameters

\subsubsection{Modeling of the Plane Surface}

The same treatment is carried out in the case of the plane surface. The attributes of the associated surface are calculated after optimization of the distance $y_{i}$ Fig. (6).

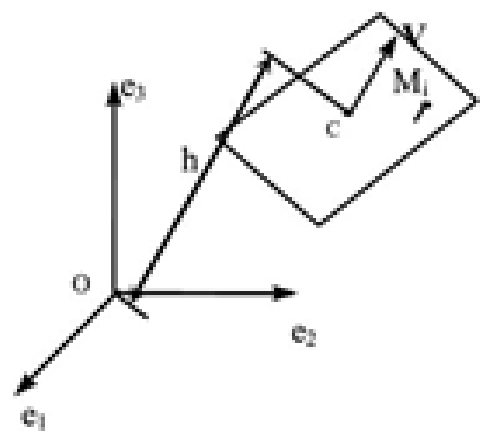

Fig. (6). Modeling of a plane. 
In the same manner as for the cylinder, the plane is defined by its normal vector $\mathbf{v}$ characterized in a cylindrical coordinate system and the distance $h$ between the plane and the center $\mathrm{O}$ of the measurement reference frame.

It has to be pointed out, that with the least squares method, the center of gravity $\mathrm{C}$ of the measured coordinates belongs to the optimized surface. The defect $y_{i}$ between the measured point and the associated surface is given by:

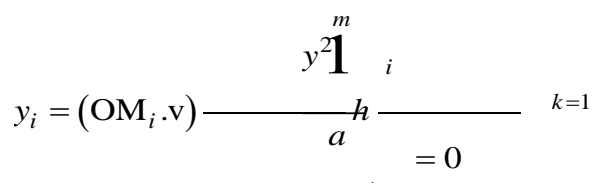

With: $\mathrm{i}=1$ to $\mathrm{m}$ digitized points

$\mathrm{a}_{\mathrm{j}}$ are the parameters of optimization

\section{CONTROL PRODEDURE}

To be inline with the philosophy of « concurrent engineering », it is possible now to suggest a methodology for the control, for that, two possibilities have been explored:

- Control incorporated transfer flows of Type I and II

- Control or pairing with associated surfaces (level1)

The proposed control is called of level 1 since it integrates the same doubt because it is based on a set of points sampled onto the real surface. The necessary inputs for the control procedure are a file containing all the points $\mathrm{Mi}$ of the measured surfaces in the measurement referential system. The data of the assembled surfaces are necessary.

\subsection{Building of the Reference System Linked to the Measured Part}

The control starts with an acquisition stage of real surfaces. To this intent a «Trimesure $1004^{\mathrm{E}}$ » coordinate measure machine (CMM) equiped with the latest release of the «Metromec » software, has been used. A pre-processing of the data retrieves a points file in the VDA format given by the machine software Fig. (7).

At the step of analyzing the measurements, the vectors $\mathbf{v}$ and the characteristic points $\mathbf{C}$ of the fitted surfaces are determined, in the measurement coordinate system. These attributes of the fitted surfaces are obtained through our non linear least squares optimization. For that purpose, the difference $y_{i}$ between a given measured point and the associated surface is computed without any linearization.

The radius of the extreme fit cylinder is obtained by the sum of the radius $\mathrm{R}$ of the best fit surface and the maximal distance $y_{i}$ between the best fit surface and the digitized points. The choice of maximal distance depends on the type of surface which is considered Fig. (8). When the surface is a shaft, the maximal distance increases the value of the best fit radius. For the hole, the radius of the extreme fit surface decreases.

After the step of treatment, the two cylinders closest to the maximum matter envelop (extreme fit) are known. These information permits to define the hierarchy of surfaces to be assembled [20].

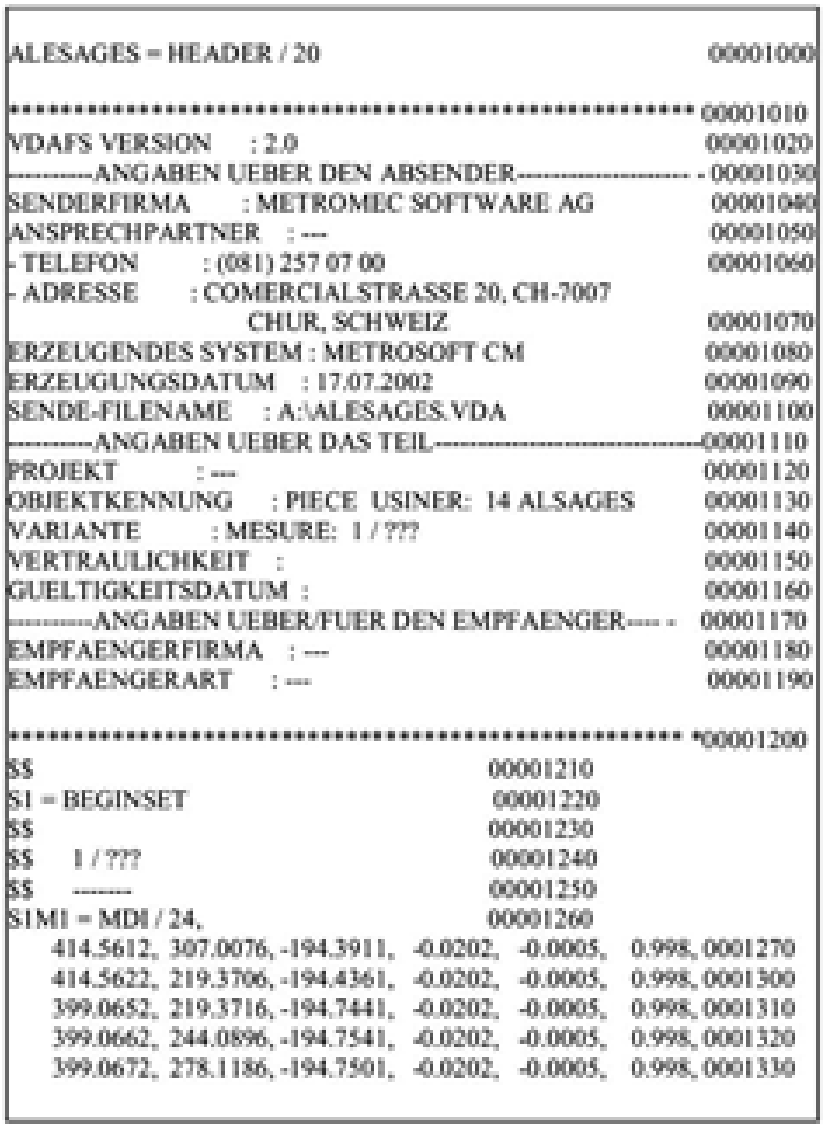

Fig. (7). Beginning of the VDA format file.

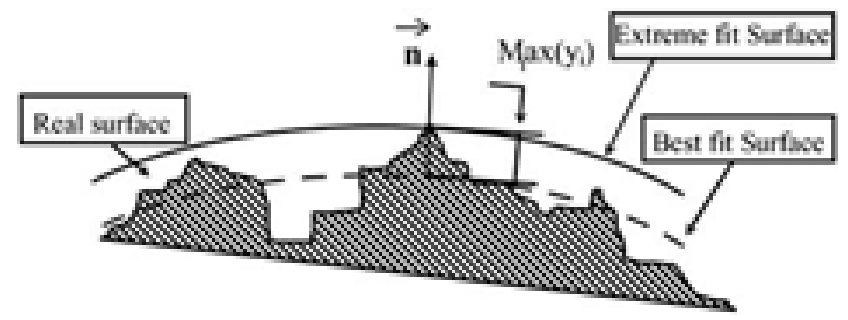

Fig. (8). Extreme fit surface for the shape of a shaft.

- $\mathrm{C}_{1}$ will be the cylinder which is closest to the maximum matter condition;

- $\mathrm{C}_{2}$ the cylinder which is closest to the cylinder $\mathrm{C}_{1}$;

- $\mathrm{C}_{3}$ the last.

After this stage, the coordinate system of the surface group is built according to the classification define before. In the present case, the primary axis (the axis $\mathrm{Z}_{\mathrm{FG}}$ ) is the axis associated with $\mathrm{C}_{1}$ Fig. (9).

The second axis is supplied by the vector $\mathrm{O}_{1} \mathrm{O}_{2}\left(\mathrm{O}_{1}, \mathrm{O}_{2}\right.$ are the points of $\mathrm{C}_{1}, \mathrm{C}_{2}$ which intercept the theoretical plane $\mathrm{P}$ normal to $\mathrm{C}_{1}$ and containing the highest point of $\mathrm{P}_{1}$ ) Fig. (10). The third axis is defined by the vectorial product of the two previous vectors. The origin of the axis of the Functional 

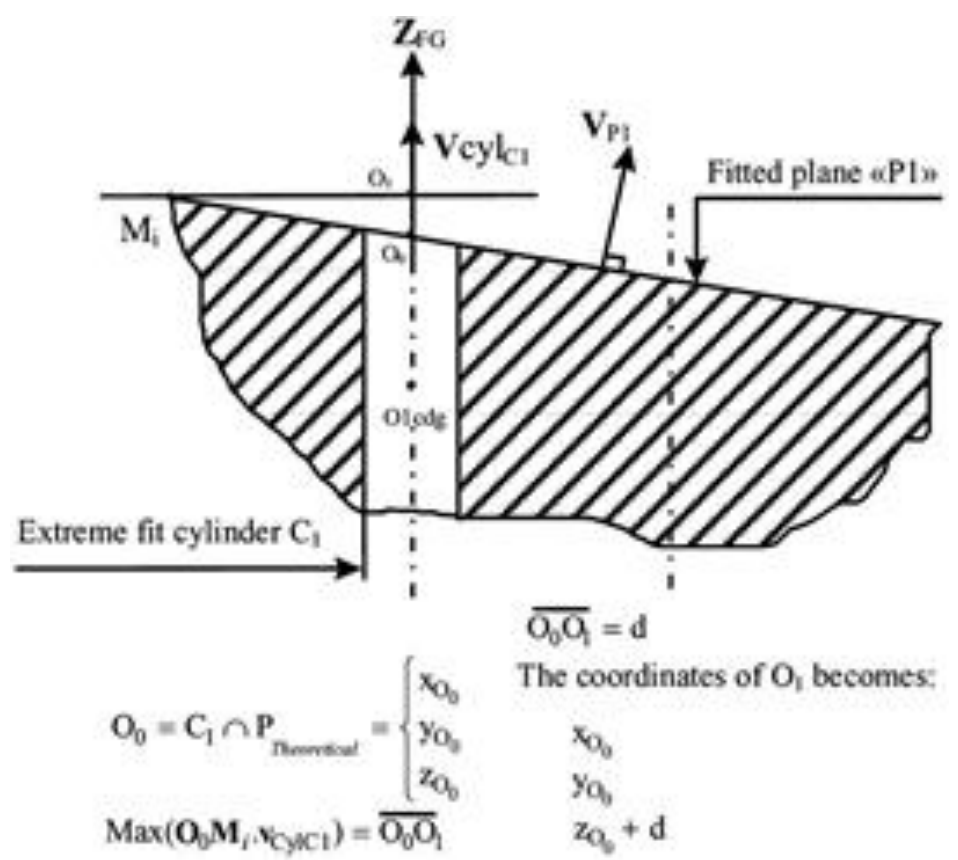

Fig. (9). Determination of the first axis.

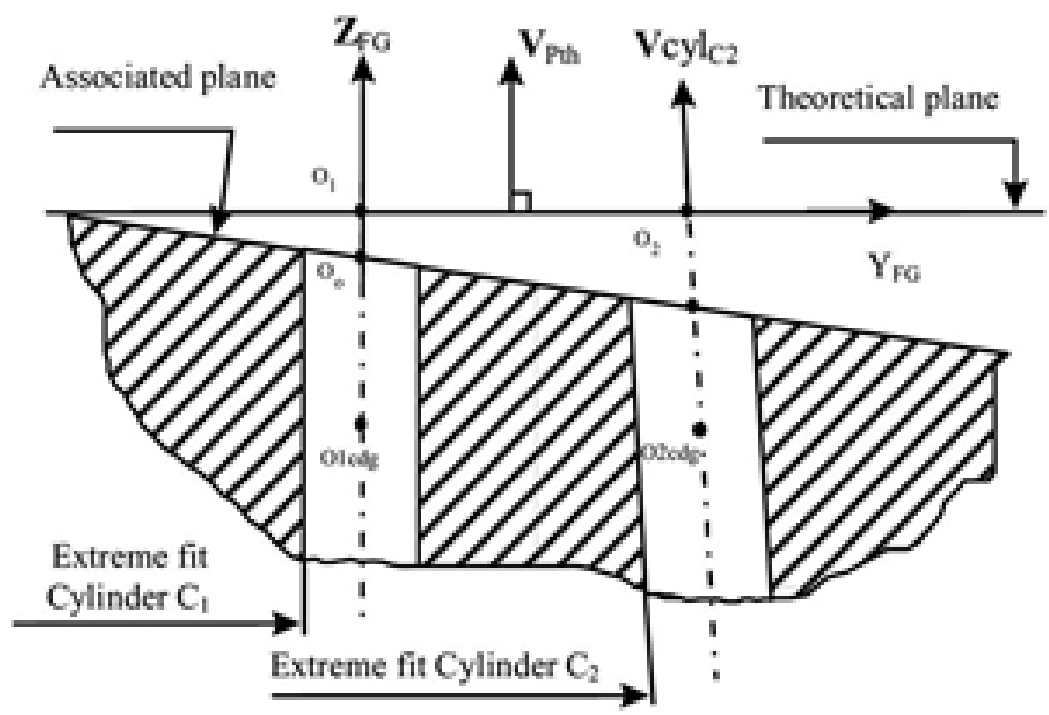

Fig. (10). Determination of the second axis.

Group $\left(\mathrm{O}_{\mathrm{FG}}\right)$ is the intersection of axis $\mathrm{Z}_{\mathrm{FG}}$ with a theoretical plane.

The configuration of Fig. (11) is obtained:

All the vectors of surfaces and the characteristic points $\left(\mathrm{C}_{\mathrm{i}}\right)$ are then carried to the reference frame of the group surfaces. At this step, the control starts with the comparison of each diameter to its admissible limits.

\subsection{Building of the Reference System Linked to the Gauge}

The build information is proposed by the geometric specification imposed by the designer. The gauge surfaces are in maximum matter condition.

\subsection{Control Procedure}

In the first step of the control procedure, it is necessary to compare the diameters of extreme fit cylinders with their acceptable limits Fig. (12). The second control operation consists of finding an assembly case for which the intersection between the ends/extreme fit of the associated cylinders of both surface groups with the cylinders of the virtual gauge does not exist.

The search for the assembly case, made possible by the freedom space, is operated by statistical exploration of its domain. Two cases arise:

- No intersection, then the assembly is possible, 


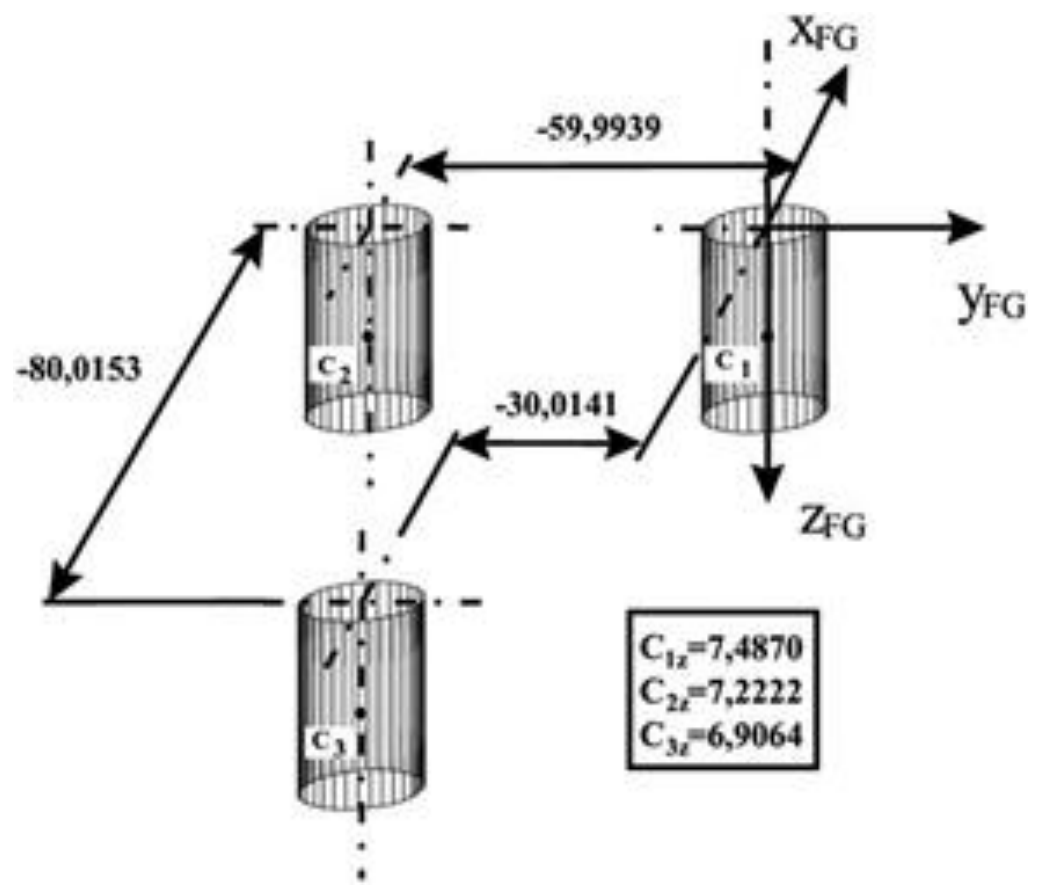

Fig. (11). Configuration obtained after optimization.

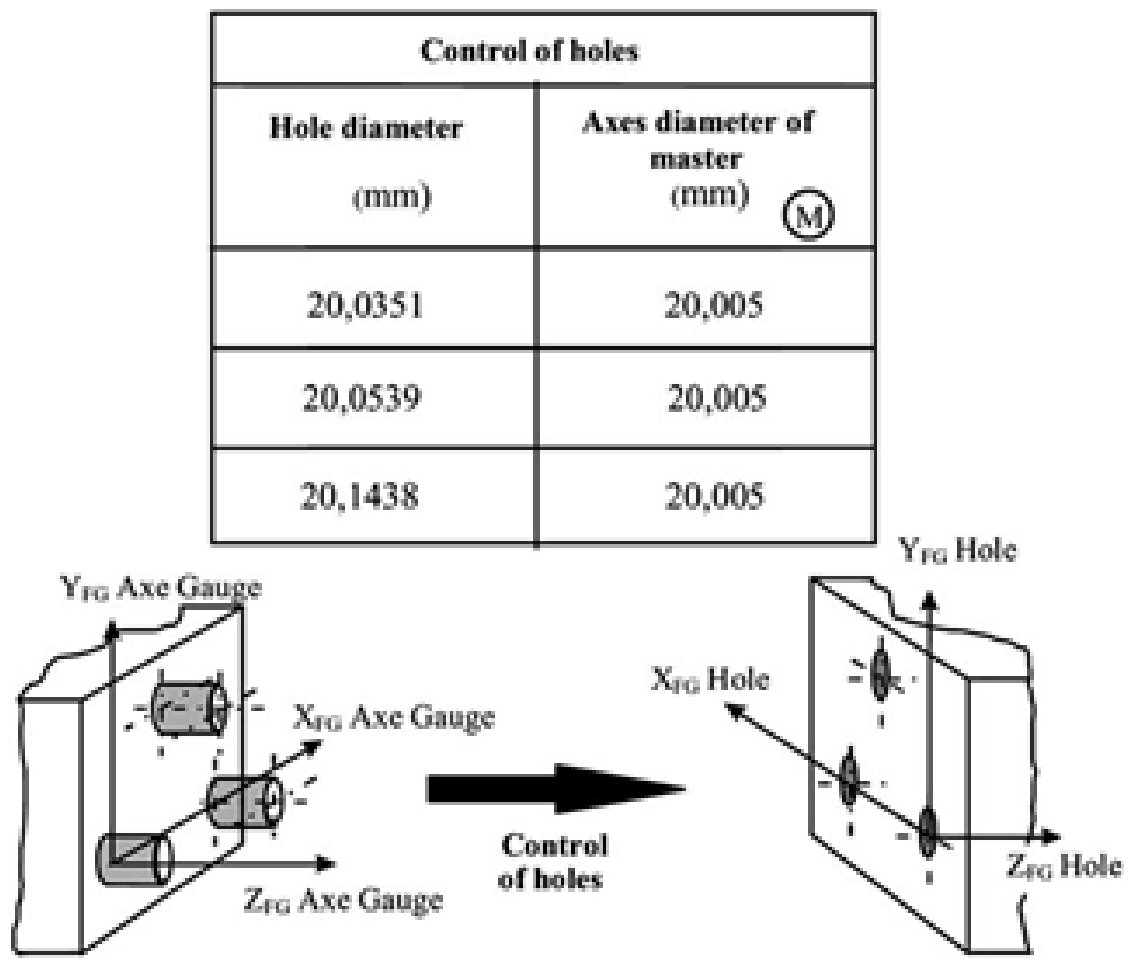

Fig. (12). Control of holes.

- Existence of an impossible intersection thus assembly.

The procedure of assembly imposes a hierarchy between assembled surfaces.

The first case does not pose a problem. In the second case, the statistical exploitation of the space of freedom, enables us to move the reference frame of the virtual gauge compared to that of the group of surfaces until there is a possibility of assembly Fig. (13).

This space of freedom is a function of the existing clearance $\mathrm{J}_{1}$ between $\mathrm{C}_{1}$ and $\mathrm{C}_{1 \text { Gauge }}$ is assumed to be lower than the clearance $\mathrm{J}_{2}$ between $\mathrm{C}_{2}$ and $\mathrm{C}_{2 \text { Gauge, and it's }}$ generated in a three-dimensional volume modelled by the 5 


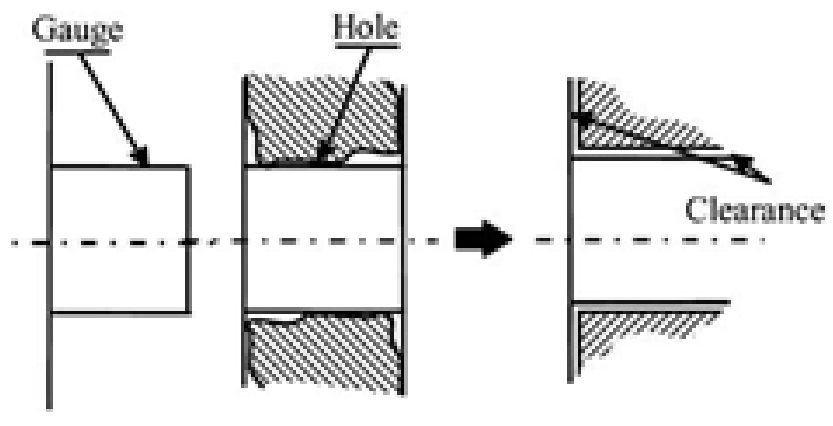

Fig. (13). Assembly gauge-hole.

parameters of the interface torsos (2 translations and 3 rotations).

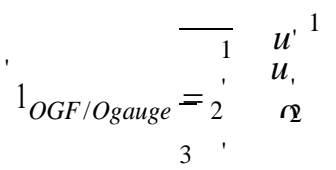

The study of the connection Fig. (14), shows that positioning in translation is constrained by the clearance $\mathbf{J}_{1}$ and the precision of indexing in rotation depends primarily on the clearance $J_{2}$, to respect this configuration the value of the clearance $J_{1}$ is lower or equal to the value of the clearance $\mathrm{J}_{2}$.

In our case, the clearance $J_{1}$ is equal to the difference between the two radius $R_{1}$ (radius of associated holes) and $r_{1}$ (radius of cylinders gauge). In the same way for $\mathrm{J}_{2}$ :

$J_{1}=2 .{ }^{\prime} R_{1} r$ and $J_{2}=2 . R_{2}^{\prime} r \quad{ }^{\prime}$

whith :

$R_{1}, R_{2}$ :the radius of associated holes

$r_{1}, r_{2}$ :the radius of cylindre gauge

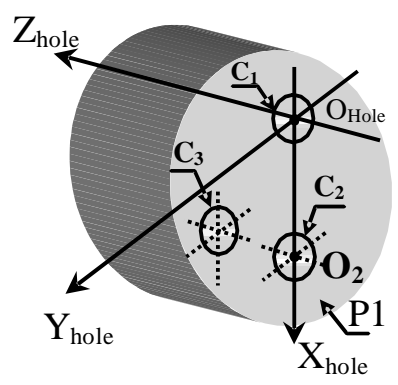

That can be simplified by representing this configuration by the presence of two axis inside two cylinders having a diameter equal to the values of the clearances Fig. (15).

Writing the extreme conditions with the limits imposed by clearances, give us the following equations:

$$
\begin{aligned}
& \sqrt{\left[u_{1}^{\prime}\right]^{2}+\left[u_{2}^{\prime}\right]^{2} \quad 0,5 . J_{1}} \\
& \sqrt{\left[u_{1}^{\prime} 1 L_{z} \cdot \frac{\left.{ }^{\prime}\right]^{2}+\left[u_{2}^{\prime}+L_{z}{ }^{\prime}\right.}{1}\right]^{2}} \quad 0,5 . J_{1}
\end{aligned}
$$$$
\sqrt{\left[u_{1}^{\prime}\right]^{2}+\left[u_{2}^{\prime}+L_{x} \cdot{ }^{\prime}\right]^{2} \quad 0,5 . J_{2}}
$$

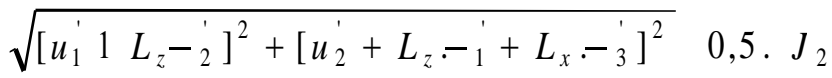

The displacement authorized by the interface with available clearance $J_{1}$ and $J_{2}$ between the surfaces group with the cylinders and virtual gauge is defined Fig. (15). It's are given by the following equations:

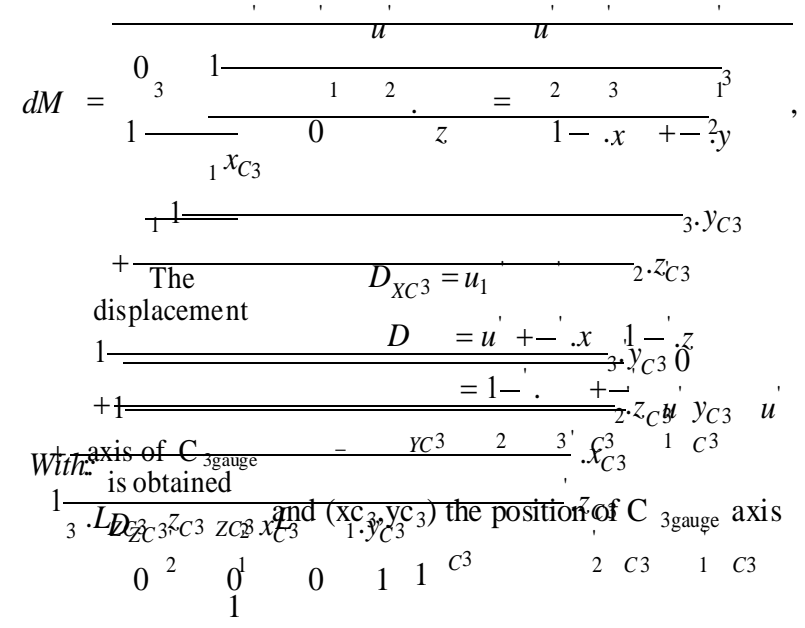

Fig. (14). Assembly possibility.

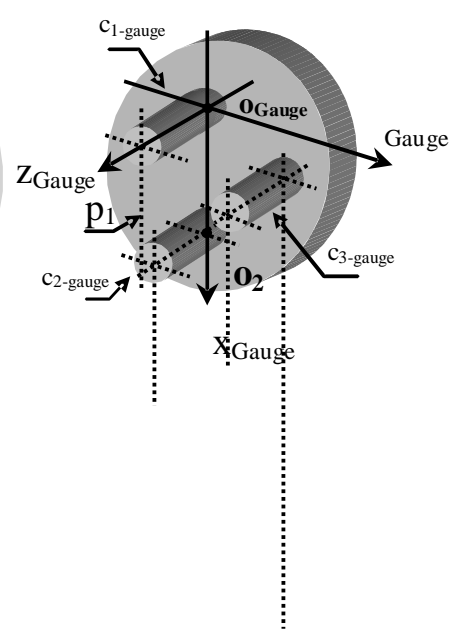



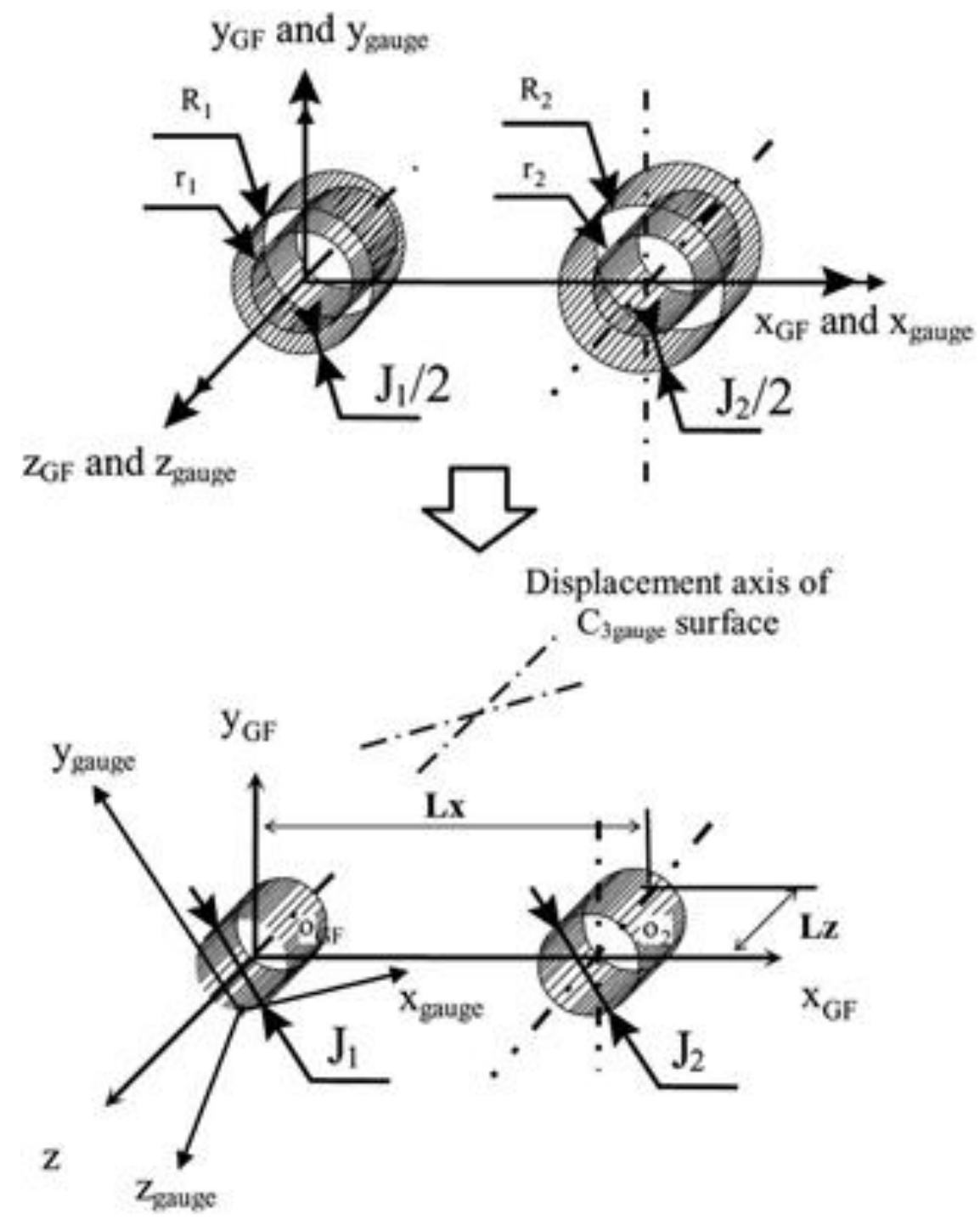

Fig. (15). Topology parameters.

Confronted with the complexity implicit equations which are difficult to solve analytically two treatments may be used:

- An analytic treatment: The implicit equations are very complex and difficult to solve

- An statistic treatment by a Monte Carlo method

The Monte Carlo simulation method has been published in many papers and books. It is well adapted to solve problems which depend on a great number of independent variables. In our single example, the number of internals and externals variables is greater than 10 Fig. (16).

Generally, all of the items of interest in these situations can be written in the form of equations, usually of considerable complexity and rarely amenable to explicit solution. The usual approach for handling these situations is the simulation.

The Monte Carlo method impose the knowledge of the distribution laws of each independent variable. It should be evident that although the mathematical definition of randomness can be precise, the practical definition (Rnd function) depends on the software and the application. A number of criteria have been proposed for judging the quality of these generators (serial correlations of the pseudo random generator).

Such Rnd function should have has some qualities:

- Stability. The generator should pass all the statistical tests and have an extreme long period.

- Efficiency. Its Execution should be rapid and the storage requirement minimal

- Repeatability. A fixed starting condition (Randomize function) should generate the same sequence.

- Simplicity. The algorithm should be easy to implement and use.

A demonstration software has been developed. At each iteration, a new position of the axis of cylinder $\mathrm{C}_{3}$ (or $\mathrm{C}_{3 \text { gauge }}$ ) is calculated. After a great number of simulations, all the 


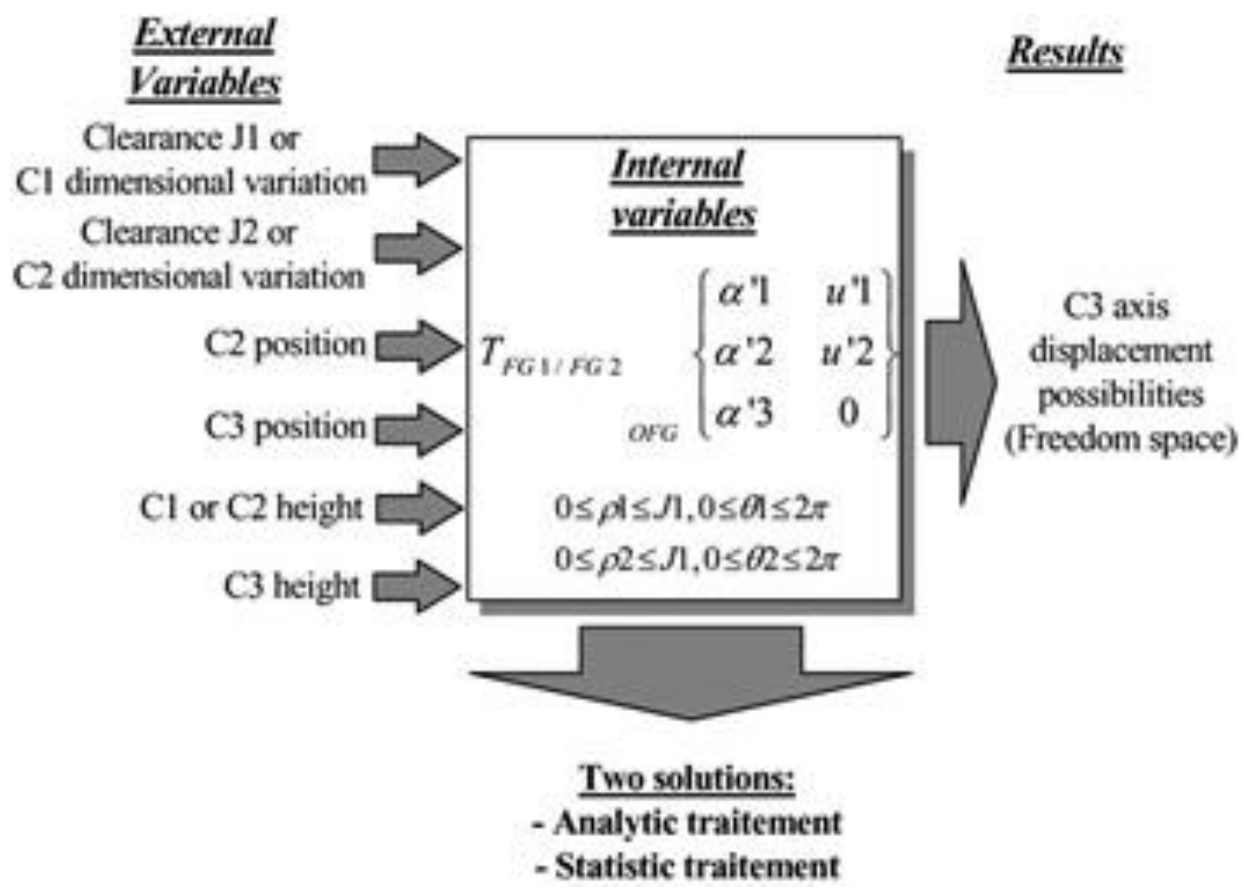

Fig. (16). External and internal variables.

possible displacements of these axis $\mathrm{C}_{3}$ (or $\mathrm{C}_{3 \text { gauge }}$ ) can be plotted Fig. (17). The boundary of the domain which is thus obtained represents the freedom space of $\mathrm{C}_{3}$ (or $\left.\mathrm{C}_{3 \text { gauge }}\right)$.

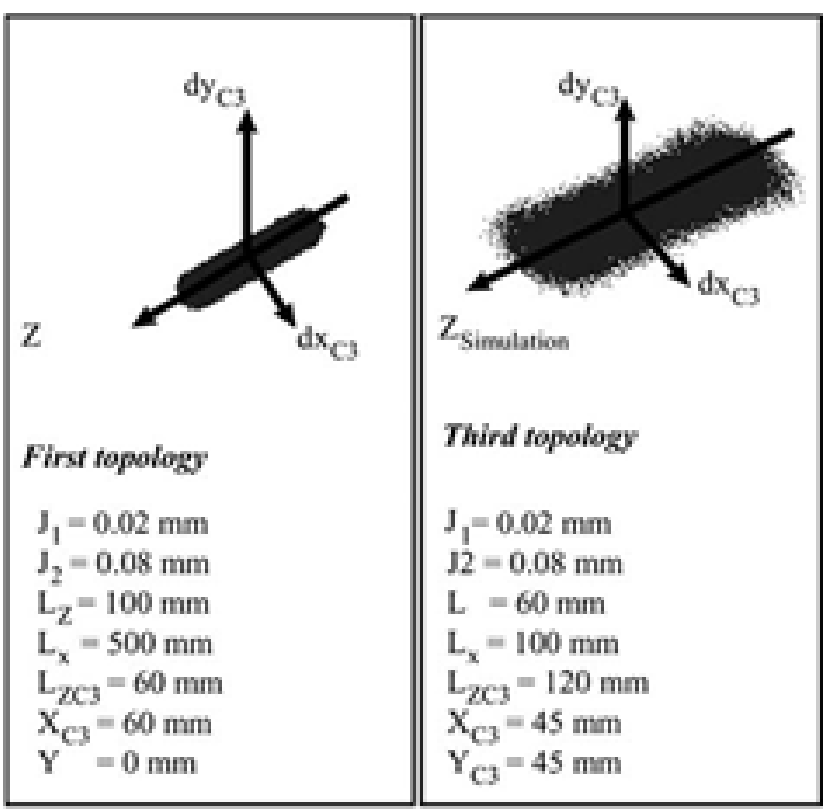

Fig. (17). Some simulations results.

The research of the case of assembly, made possible by the space of freedom, is carried out by a statistical exploration of its field. This research does not present any difficulty of calculation, because simulation information of the assembly is of a great flexibility in use.
This methodology makes pairing possible between two Functional Groups represented by their mathematical images/extreme fits. We can either perform control between the computer gauge and the associated surfaces of one Functional Group or between the associated surfaces of two Functional Groups.

\section{CURRENT \& FUTURE DEVELOPMENTS}

The maximal material condition has an extent use in the geometric specification of the mechanical parts. It permits to specify the fitting condition. When the datum references or the specification use this requirement, it is very difficult to verify the geometrical specification.

This paper focuses on the verification of this type of specification by virtual gauge. This proposition is based on the freedom space. It permits to control the displacement possibilities of the gauge onto the part. The determination of the freedom space necessities the accuracy of the surface parameters.

The no linear calculation permits to obtain theses parameters directly in the global reference frame associated at the coordinate measurement machine. With theses parameters it is possible to calculate the maximal material states of the surfaces. Theses indications permit to define the clearances between the virtual gauge and the extreme fits of the surfaces. The values of the clearances permit to calculate the freedom space. It defines the displacement possibilities of the gauge onto the part. The displacements permit to assembly the gauge with the part when the assembly is possible. This procedure is never possible with the requirement of the minimal material condition. In this case only the use of virtual gage is possible. 


\section{REFERENCES}

[1] Grupp, G., Kammleiter, B.: US20080177491 (2008)

[2] Wen-Yuh J, Chien-Hong L, Cha'o-Kuang C. The min-max problem for evaluating the form error of a circle. Measurement 1999; 26: 273-282.

[3] Jiing-Yih L, Wen-Der U. Reconstruction of surfaces of revolution from measured points. Comput Industry 2000; 41: 147-161.

[4] Sprauel JM, Linares JM, Bourdet P. Contribution of nonlinear optimization to the determination of measurement uncertainties. In Proc of the seventh CIRP seminar on computer-aided tolerancing, Cachan, France 2001; 285-291.

[5] Antony GT, Anthony HM, Bittner B, et al. Reference software for finding Chebyshev best fit geometric elements. Precision Eng 1996; 197: 28-36.

[6] Bourdet P, Mathieu L, Ballu A. The concept of the small displacement torsor in metrology, Advanced Mathematical Tools in Metrology II, World Scientific Publishing Company 1996; 110122.

[7] Goch G. Gear metrology, Keynote papers. Ann CIRP 2003; 52 (2): 1-37.

[8] Jackson, D., Shroff, H., Christian, D.J., Glickman, S.: WO0146909 A1 (2001).

[9] Asme Y. Dimensioning and tolerancing. The American Society of Mechanical Engineers, New York 1994.
[10] Boukebbab S, Bouchenitfa H, Boughouas H, Linares JM. Applied iterative closest point algorithm to automated inspection of gear box tooth. Int J Comput Indus Eng 2007; (52), 162-173.

[11] Etesami F. Position tolerance verification using simulated gaging. Int J Robot Res 1991; 10 (4): 358-370.

[12] Robinson DM. Geometric tolerancing for assembly with maximum material parts, in: CIRP Seminar on Computer Aided Tolerancing. Toronto, Canada, April 1997.

[13] Dantan JY, Ballu A. Assembly specification by gauge with interna] mobilities: A specification semantic deduced from the tolerance analysis. J Manuf Syst 2002; 2(3): 218-235.

[14] Mailhe J, Linares JM, Sprauel JM, Bourdet P. Geometrical checking by virtual gauge, including measurement uncertainties. CIRP Ann Manuf Technol 2008; 57 (1): 513-516.

[15] Sturtevant, J.L., Gu, Y.: US20087349752B1 (2008)

[16] Linares JM, Boukebbab S, Sprauel JM. Parametric tolerancing, $6^{t}$ CIRP Int seminar on Comp Aided Toleranc. University of Twente, Enschede, The Netherlandes, Mars 1999; 167-176.

[17] Bourdet P, Clement A. A study of optimal-criteria identification based on the small displacement screw model. Ann CIRP 1988; 37 : 503-506.

[18] Sprauel JM, Linares JM, Bourdet P. Contribution of non linear optimization to the determination of measurement uncertainties, selected papers book of the Computer Aided Tolerancing CIRP, Geometric specification and verification: Integration of functionality ed Pierre Bourdet et Luc Mathieu, Kluwer Academic Publisher 2003; 237-244.

[19] Pairel E. Three-dimensional verification of geometric tolerances with the "Fitting Gauge" Model. J Comput Inform Sci Eng 2007; 7(1): 26.

[20] ISO TS 17450-1, Geometrical product specification, General concepts, Part 1: Model for geometric specification. 